Int. J. Electrochem. Sci., 15 (2020) $4884-4899$

\title{
Electrocatalytic Activity of Graphene Oxide/Metal Organic Framework Hybrid Composite on Hydrogen Evolution Reaction Properties
}

\author{
Mogwasha D. Makhafola ${ }^{1}$, Kabelo E. Ramohlola ${ }^{1}$, Thabiso C. Maponya ${ }^{1}$, Thabang R. Somo ${ }^{1}$, \\ Emmanuel I. Iwuoha ${ }^{2}$, Katlego Makgopa ${ }^{3}$, Mpitloane J. Hato ${ }^{1,4, *}$, Kerileng M. Molapo ${ }^{2}$, \\ Kwena D. Modibane ${ }^{1, *}$ \\ ${ }^{1}$ Nanotechnology Research Lab, Department of Chemistry, School of Physical and Mineral Sciences, \\ University of Limpopo (Turfloop), Sovenga 0727, Polokwane, South Africa \\ ${ }^{2}$ SensorLab, Chemistry Department, University of the Western Cape, Cape Town, South Africa \\ ${ }^{3}$ Department of Chemistry, Faculty of Science, Tshwane University of Technology (Acardia Campus), \\ Pretoria 0001, South Africa \\ ${ }^{4}$ Department of Environmental Sciences, College of Agriculture and Environmental Sciences, \\ University of South Africa, Florida Science Campus, Johannesburg 1710, South Africa

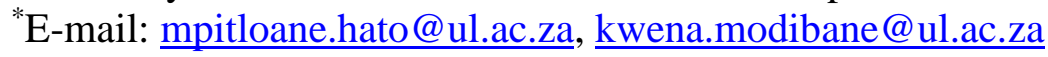

doi: $10.20964 / 2020.06 .10$

Received: 30 November 2019 / Accepted: 22 February 2020 / Published: 10 May 2020

In this work, the electrocatalytic activity of graphene oxide (GO) and its composite with metal organic framework (MOF) towards electrochemical hydrogen evolution reaction (HER) was studied. GO/MOF composite was synthesized by impregnation route and characterized using X-ray diffraction (XRD), Fourier transform infrared spectroscopy (FTIR), simultaneous thermal analysis (STA), scanning electron microscopy/energy dispersive spectroscopy (SEM/EDS) and high-resolution transmission electron microscopy/energy dispersive X-ray spectroscopy (HR-TEM/EDX). STA analysis showed an enhancement of the thermal stability of the GO/MOF composite compared to the pristine GO and MOF. SEM/EDS and HR-TEM/EDX confirmed the presence of octahedral structure of MOF in the GO sheetlike structure and elemental composition of the synthesized compounds. The performance of the proposed electrolytic system for electrochemical HER by Tafel parameters and turn over frequencies (TOFs) showed a huge increment of $\mathrm{H}_{2}$ production in the composite through the Volmer reaction together with either of the two mechanisms in HER. This indicated that the addition of GO/MOF in the electrolytic system possessed better catalytic characteristics with enhanced TOFs, which may open a new way for hydrogen technology via HER enhancement.

Keywords: electrocatalyst; graphene oxide; hydrogen evolution reaction; metal organic frameworks; Tafel analysis 


\section{FULL TEXT}

(C) 2020 The Authors. Published by ESG (www.electrochemsci.org). This article is an open access article distributed under the terms and conditions of the Creative Commons Attribution license (http://creativecommons.org/licenses/by/4.0/). 